ODILE RICHARD-PAUCHET

ORCID: 0000-0001-5373-9405

Université de Limoges

odile.pauchet@unilim.fr

\title{
LES PHILOSOPHES DE PALISSOT (1760) : LA QUERELLE ET LES PASSIONS ÉPISTOLAIRES QUI S'ENSUIVIRENT
}

On se souvient aujourd'hui de Charles Palissot (1730-1814) surtout pour les sarcasmes qu'il reçut de Diderot dans Le Neveu de Rameau, où il est présenté par Rameau lui-même comme un fidèle de la «ménagerie de Bertin », ce petit cercle de plumitifs misérables reçus par le financier et sa maîtresse, l'actrice $\mathrm{M}^{\text {lle }}$ Hus :

Nous avons, comme vous savez, la compagnie la plus nombreuse et la mieux choisie [...]. Tous les poètes qui tombent, nous les ramassons ; nous eûmes Palissot, après sa Zarès, Bret après Le Faux généreux; tous les musiciens décriés, tous les auteurs qu'on ne lit point, toutes les actrices sifflées, tous les acteurs hués, un tas de pauvres honteux, plats parasites à la tête desquels j'ai l'honneur d'être, brave chef d'une troupe timide [...]. Jamais on ne vit ensemble tant de bêtes tristes, acariâtres, malfaisantes et courroucées [...]. C'est là que le plan de la comédie des Philosophes a été conçu1.

Cette évocation sans pitié révèle chez Diderot une haine non éteinte, qui surprend chez le célébrissime Encyclopédiste, s'agissant d'un si médiocre adversaire. Certes la postérité, comme il le prévoyait génialement, a fait aujourd'hui un tri judicieux. Mais la colère sensible dans le réquisitoire poétique que constitue Le Neveu de Rameau, montre que la certitude d'être reconnu un jour au détriment du vermisseau Palissot, était loin d'être présente au moment de l'écriture (une écriture étalée pourtant sur presque 15 ans : 1760-1773). Comme le souligne avec émotion Sylviane Albertan-Coppola, dans son article «Les Anti-philosophes dans Le Neveu de Rameau » : "C'est la main tremblante que l'on imagine Diderot écrivant Le Neveu de Rameau. Tout donne à penser que Diderot, malgré son parti-pris

1 D. Diderot, Le Neveu de Rameau, J. Fabre (dir.), Droz, Genève 1963, p. 57. 
apparent, de détachement méprisant, dont le philosophe se fait l'écho [...] a été très blessé par la comédie de Palissot $»^{2}$. Cette réplique ad hominem si violente, même et surtout dans un texte posthume comme Le Neveu de Rameau, mérite qu'on revienne sur les circonstances de la représentation des Philosophes de Palissot, pour comprendre à quel point cette comédie plutôt creuse a pu constituer un véritable traumatisme tant pour le directeur de l'Encyclopédie, personnellement attaqué, que pour les Encyclopédistes qui l'entourent à l'époque, enfin pour la société intellectuelle parisienne, française et européenne en général ${ }^{3}$. On montrera comment l'onde de choc a pu se propager à plusieurs niveaux : d'abord par le manuscrit en circulation, les répétitions des comédiens et la représentation ellemême (le 3 mai 1760) ; ensuite par la publication, pendant tout l'été, de diverses brochures ennemies ou amies ; enfin par le retentissement épistolaire auquel ce texte donna lieu, notamment grâce à Voltaire, personnage admiré de Palissot, mais dont les lettres souvent ambiguës furent à la fois un remède et un poison à cet événement médiatique si funeste.

$$
* * *
$$

Charles Palissot de Montenoy, à en croire les critiques qui se sont penchés sur sa carrière ${ }^{4}$, n'est pas sans rappeler le personnage de Voltaire lui-même (le génie en moins), par sa précocité et son ambition. Né à Nancy, il fut protégé par un autre Lorrain, le comte de Stainville, futur duc de Choiseul, dont le père aurait eu le père de Palissot pour homme de confiance ${ }^{5}$. Auteur dramatique précoce, il ambitionne très vite de devenir, à 21 ans, le nouveau Corneille avec sa tragédie Zarès, jouée le 3 juin 1751 à la Comédie-Française (mais qui n'aura que 3 représentations). Sans se laisser abattre, il tente alors la comédie et fait jouer Les Tuteurs à la Comédie-Française le 2 septembre 1754, en deuxième partie de la Rodogune de

2 S. Albertan-Coppola, «Les Anti-philosophes dans Le Neveu de Rameau», Cahiers Textuel 11, 1992, pp. 33-41. Elle cite à la suite cet autre passage du Neveu de Rameau : « [...] un homme souillé de toutes sortes d'infamies, qui par passe-temps fait abjurer la religion à son ami, qui s'empare du bien de son associé ; qui n'a ni foi, ni loi, ni sentiment ; qui court à la fortune, per fas et nefas ; qui compte ses jours par ses scélératesses ; et qui s'est traduit lui-même comme un des plus dangereux coquins, impudence dont je ne crois pas qu'il y ait eu dans le passé un premier exemple, ni qu'il y en ait un second dans l'avenir » (D. Diderot, op. cit., p. 69). Voir aussi de S. Albertan-Coppola, « Entre la correspondance de Diderot et Le Neveu de Rameau : les Antiphilosophes ", Épistolaire 41, Champion, Paris 2015, pp. 175-186.

${ }^{3}$ Sur la pratique de la satire et de l'attaque ad hominem au théâtre, voir O. Ferret, « Mises en scène satiriques des Encyclopédistes : autour de la querelle des philosophes », [dans :] Le Philosophe sur les planches. L'image du philosophe dans le théâtre des Lumières :1680-1815, P. Hartmann (dir.), Presses de l'Université de Strasbourg, Strasbourg 2003, pp. 113-128.

4 Voir T.-J. Barling, « Introduction » à son édition critique des Philosophes, University of Exeter, Exeter 1975, p. V.

5 Voir E. Showalter, « Madame de Graffigny, Palissot et Les Philosophes », Recherches sur Diderot et sur l'Encyclopédie 20, 1997, p. 114. 
Corneille. Là, un succès d'estime fait qu'elle sera reprise à l'automne. L'intrigue assez légère traite des amours de Damis avec Julie : celui-ci, pour épouser sa belle, doit convaincre ses trois tuteurs et en adopter les manies : l'un est antiquomane, l'autre un nouvelliste, le troisième un voyageur impénitent. Ce goût pour la satire des mœurs françaises se retrouvera dans Les Philosophes. Palissot ne doute plus en effet d'être devenu le Molière de son siècle : «Tout le monde veut que je fasse des comédies ; on soutient que c'est mon genre [...], on a trouvé que j'avais travaillé dans le vrai, dans le bon comique, celui de Régnard, et quelquefois celui de Molière [...]. C'est aussi la seule chose dont je m'applaudisse », écrit-il modestement à Jacob Vernes le 5 octobre 1754. Dans le Discours préliminaire très soigné qui accompagne la parution de cette comédie, dédiée à la comtesse de La Marck, il regrette la décadence du théâtre comique en France, et prétend que « ce sont surtout les mœurs bourgeoises que l'on doit peindre au Théâtre ${ }^{6}$. Il existe donc bien chez Palissot une ambition littéraire, un projet poétique tourné vers la refondation de la comédie bourgeoise à la française, dont la vocation serait tout simplement de moquer les nouveaux ridicules en restant fidèle à la plus pure tradition de la pièce en alexandrins, celle qui respecte les unités de lieu, de temps et d'action, les règles de vraisemblance et de bienséance. À 25 ans, le succès lui monte à la tête : soutenu par le critique Fréron, bête noire de Voltaire, il est protégé personnellement par Choiseul, futur ministre des Affaires Étrangères, qui le fait nommer en 1755 à la Recette générale du Tabac d'Avignon, le mettant provisoirement à l'abri du besoin. Cette année-là, il rend visite à Voltaire à Genève et se charge, à la demande du roi Stanislas qui l'a nommé Académicien, d'un divertissement pour les fêtes publiques de Nancy destinées à l'inauguration de la statue de Louis XV, beau-père du roi. Se sentant invincible, il a l'audace de s'attaquer, dans ce divertissement, à de nouvelles cibles "médiatiques ", en particulier à Rousseau. La pièce, intitulée Le Cercle ou les Originaux, jouée et imprimée à Nancy à l'automne 1755, $\mathrm{y}$ met en scène « une femme savante, un poète infatué »" soit Voltaire et $\mathrm{M}^{\mathrm{me}} \mathrm{du}$ Châtelet, sous les traits de «M. du Volcan, écrivain, et d'Araminthe, bas-bleu »; ainsi qu'un philosophe avide de gloire, Blaise-Nicodème le Cosmopolite, plus préoccupé « de soutenir des "paradoxes bizarres" que de découvrir des "vérités neuves" ${ }^{8} . .$. Le comte de Tressan, soutenu par d'Alembert, demande l'interruption de la pièce et l'expulsion de Nancy de Palissot, qu'il n'obtiendra pas, tant sont solides les soutiens du jeune auteur, notamment celui de la princesse de Robecq, maitresse probable de Choiseul.

${ }^{6}$ Ch. Palissot de Montenoy dans ses Mémoires (CEuvres, t. I, Liège 1777, p. 77). Sur la technique « moliéresque » de Palissot, voir aussi C. Bonfils, " Charles Palissot et la tradition moliéresque. L'Homme dangereux », Revue d'Histoire Littéraire de la France 6, novembre-décembre 1997, pp. 1008-1023.

7 Ch. Palissot de Montenoy, La comédie des "Philosophes » et autres textes, H. Duranton (dir.), présentation d'O. Ferret, Publications de 1'Université de Saint-Étienne, coll. « Lire le XVIII ${ }^{\mathrm{e}}$ siècle », Saint-Étienne 2002, p. 7 (Préface).

8 Ch. Palissot de Montenoy, Les Philosophes, T.-J. Barling (dir.), 1760, p. IX. 
Cet épisode, qui aurait dû avertir Palissot du danger de s'en prendre à des sujets sensibles, fouette au contraire son orgueil et le convainc d'adhérer pleinement à la cause anti-philosophique. Dans ses Mémoires, il se flattera d'ailleurs hypocritement de s'attaquer, tel Aristophane, toujours aux idées, jamais aux hommes. Il publie en effet en 1757 Petites Lettres sur de grands philosophes, où il s'en prend pêle-mêle à travers leurs œuvres, à divers auteurs de la cause encyclopédique, notamment au Fils Naturel de Diderot, dont il est incapable d'apprécier la nouveauté, et qu'il préfère taxer de plagiat à l'égard de Goldoni.

Cette année-là, le contexte devient de plus en plus défavorable aux philosophes : en janvier 1758 a lieu l'attentat de Damiens contre la personne de Louis XV ; et l'article Genève de d'Alembert alarme les autorités tant françaises que suisses. En 1758, d'Alembert se retire alors avec fracas de l'Encyclopédie ( J'y renonce absolument »), mais Helvétius publie imprudemment son ouvrage De l'Esprit, qui défend avec Hobbes l'importance de l'intérêt dans la conduite humaine, et qui sera brûlé en place publique. En 1759, le privilège de l'Encyclopédie est alors révoqué par arrêté royal.

C'est ce climat qui encourage Palissot à frapper un grand coup sur ce clan affaibli contre lequel il a accumulé tant de ressentiment (notamment dans l'affaire des fausses dédicaces, placées par Diderot et Grimm en tête de traductions de Goldoni, et adressées à ses deux protectrices, $\mathrm{M}^{\text {mes }}$ de Robecq et de La Marck). Est-ce alors, comme le prétend Diderot, chez le financier Bertin et la petite Hus " que le plan de la comédie des Philosophes a été conçu », ou bien plutôt chez Choiseul et $\mathrm{M}^{\mathrm{me}}$ de Robecq ? Il est vrai que l'actrice $\mathrm{M}^{\text {lle }}$ Hus aura le privilège d'obtenir, dans la comédie des Philosophes, le rôle de Rosalie, jeune première (rôle auquel selon Rameau son physique ne lui permettait plus de prétendre).

La première représentation de cette comédie est annoncée et orchestrée comme un événement de tout premier ordre. Le 22 mars 1760, juste avant la relâche de la Comédie-Française, le manuscrit de Palissot est lu pour approbation aux comédiens par Fréron lui-même, qui leur aurait dit (selon ce que rapporte Collé dans sa notice de la pièce $)^{9}$ que c'était une comédie « sur la réception de laquelle il serait inutile de délibérer, attendu qu'elle serait jouée malgré eux », grâce à une protection dont effectivement Fréron se vantera plus tard dans une lettre à Jacob Vernes du 20 octobre 1771.

Le 14 avril, d'Alembert, ami et correspondant privilégié de Voltaire, lui annonce, sans en connaître l'auteur, que l'on a commencé à répéter la pièce, et que tout Paris en parle : «Il ne manquait plus à la philosophie que le coup de pied de l'âne. On va jouer sur le théâtre de la Comédie françoise une pièce intitulée, les Philosophes modernes. Préville doit y marcher à quatre pattes pour représenter

9 Voir L.J. Connors, «Les Philosophes selon Charles Collé », [dans :] Charles Collé au cæur de la République des Lettres, M.-E. Plagnol-Diéval et D. Quéro (dir.), Presses universitaires de Rennes, Rennes 2013. 
Rousseau ${ }^{10}$. Cette pièce est fort protégée. Versailles la trouve admirable $»^{11}$. Voltaire, qui prévoit de faire jouer deux autres tragédies dans la même période, a donc les yeux braqués sur l'événement, qu'il va relayer abondamment dans sa correspondance. Le vendredi 2 mai, date de la représentation, la recette est énorme : 4379 livres pour 1439 spectateurs. Le 6 mai 1760, Fréron annonce dans sa gazette L'Année littéraire que

Depuis la fondation du théâtre on n'a point vu, Monsieur, un concours de monde aussi prodigieux. C'était une presse, une foule, une fureur, dont il n'y eut point d'exemple. Les ouvrages des Corneilles, des Racines, des Molières, des Crébillons et des Voltaires n'ont jamais fait autant de bruit, attiré autant de spectateurs, armé autant de cabales. Le sujet de la pièce avait excité dans Paris une fermentation générale de curiosité ${ }^{12}$.

La comédie, qui reprend le canevas des Femmes savantes de Molière, présente tout simplement une clique de faux savants (Valère-Helvétius, ThéophrasteDuclos, Dortidius-Diderot) tâchant de circonvenir une femme savante, Cydalise, afin de servir leurs intérêts financiers, et notamment de faire épouser sa fille Rosalie par Valère, le plus entreprenant des trois. Dortidius, surtout, évoque (par son anagramme latinisée) Diderot, et Cydalise peut faire penser à $\mathrm{M}^{\mathrm{me}}$ Geoffrin, la généreuse hôtesse des Encyclopédistes. Mais, selon le spécialiste English Showalter, son personnage serait beaucoup plus proche de $\mathrm{M}^{\mathrm{me}}$ de Graffigny, elle-même auteure comme Cydalise, elle-même Lorraine comme Palissot et sa protectrice pendant quelque temps, elle-même tante d'une jeune fille, Minette, qui venait d'épouser un philosophe, Helvétius ${ }^{13}$. Enfin, dans la pièce, Rousseau n'est pas oublié, puisque le valet de Cydalise, Crispin, voulant confondre les faux philosophes, finit par paraître à quatre pattes (Les Philosophes, acte III, scène IX), mangeant une laitue et se disant le disciple d'un grand philosophe, pour finalement remettre à Cydalise un billet qui permet de duper ses prétendus amis ${ }^{14}$.

La représentation est houleuse, comme le rappellera l'auteur de l'un des pamphlets anonymes qui vont suivre, Lettre d'un Original aux Auteurs très-originaux

10 Jeu de scène inspiré par la réponse fameuse de Voltaire à Rousseau, qui avait fait le tour de l'Europe : « J'ai reçu, Monsieur, votre nouveau livre contre le genre humain [...]. On n'a jamais employé tant d'esprit à vouloir nous rendre bêtes. Il prend envie de marcher à quatre pattes, quand on lit votre ouvrage » (Lettre à Jean-Jacques Rousseau, 30 août 1755, [dans :] Voltaire, Correspondance, t. IV : (1754-1757), Th. Besterman (dir.), Gallimard, Paris 1978). Ce jugement vise le Discours sur l'origine et les fondements de l'inégalité parmi les hommes, point de départ de la philosophie politique de Rousseau qui annonce déjà le Contrat social.

${ }^{11}$ En post-scriptum de la lettre de d'Alembert à Voltaire du 14 avril 1760, cité dans : Th. Besterman, A world bibliography of bibliographies and of bibliographical catalogues, calendars, abstracts, digests, indexes and the like, lettre $\mathrm{n}^{\circ} \mathrm{D} 8852$, Societas Bibliographica, Lausanne 1904-1976.

12 É.C. Fréron, L'Année littéraire, t. III, Lambert, Paris 1760, p. 214.

13 Voir E. Showalter, op. cit., pp. 123-124.

14 Il s'agit d'une lettre écrite par Valère lui-même, où celui-ci écrit tout le mal qu'il pense du livre de Cydalise, qu'il a pourtant encouragée à écrire (selon E. Showalter, c'est dans cette lettre même qu'on peut identifier l'ouvrage de $\mathrm{M}^{\mathrm{me}}$ de Graffigny, les Lettres d'une Péruvienne, voir notre note supra). 
de la Comédie très-originale des Philosophes : « le flux et le reflux convulsif du parterre » l'ont « transporté dans vingt espaces différents ${ }^{15}$. L'événement déchaîna la colère des femmes : l'une, proche des Encyclopédistes, l'actrice $\mathrm{M}^{\text {lle }}$ Clairon, reprocha violemment aux Comédiens-Français leur complicité, voire leur « collaboration ». L'autre, la princesse de Robecq, protectrice de Palissot, à l'article de la mort à cette période de sa vie et crachant le sang, se traîna jusqu'au théâtre pour y soutenir son favori.

Les passions, vivement remuées par le texte et la représentation elle-même, vont continuer de s'agiter à la faveur des libelles qui paraissent pendant les semaines qui suivent, sorte de vague médiatique qui prolonge jusqu'en septembre la fureur de la parole par celle du papier.

$$
* * *
$$

Olivier Ferret a fait un inventaire passionnant, dans son édition La comédie des «Philosophes » et autres textes, de cette vingtaine d'écrits joués et/ou publiés, qui vont occuper l'opinion publique pendant tout l'été et donner plus d'importance à la pièce qu'elle n'en mérite. C'est sans doute l'effet recherché par les autorités qui, en donnant leurs autorisations de publication, ne sont pas mécontentes de voir le public se détourner d'un " contexte politique intérieur et extérieur très défavorable ${ }^{16}$, comme il l'avait déjà fait au temps de la querelle des Cacouacs (1757). Pour le détail de ces textes, on se reportera à notre tableau ci-dessous suivi de son commentaire. Nous plaçons dans la colonne de gauche la comédie de Palissot et les textes de ses défenseurs, dans celle de droite les ouvrages de ses attaquants. Nous soulignons les titres des auteurs les plus fameux.

\begin{tabular}{|l|l|}
\hline \multicolumn{2}{|c|}{ Comédies } \\
\hline $\begin{array}{l}\text { Palissot, Les Philosophes, comédie } \\
\text { jouée le 2 mai à la Comédie-Française) }\end{array}$ & $\begin{array}{l}\text { A.-Ch. Cailleau, Les Philosophes manqués } \\
\text { (parue le 15 mai) }\end{array}$ \\
$\begin{array}{l}\text { A.-A.-H. Poinsinet, dit le Jeune, Le Petit phi- } \\
\text { losophe (jouée le 14 juillet aux Italiens) }\end{array}$ \\
$\begin{array}{l}\text { A.-Ch. Cailleau, Les Originaux, ou les fourbes } \\
\text { punis, parodie (parue le 17 juillet) }\end{array}$ \\
$\begin{array}{l}\text { L. Poinsinet de Sivry, Les Philosophes de bois } \\
\text { (jouée par les marionnettes de Cadet à Passy, } \\
\text { 20 juillet) }\end{array}$ \\
\hline
\end{tabular}

15 Ch. Palissot de Montenoy, La comédie des « Philosophes »..., O. Ferret (dir.), p. 140.

16 P.-Y. Beaurepaire, La France des Lumières : 1715-1789, chap. 8 : "L'autorité royale en question », Belin, Paris 2011, p. 491. 


\begin{tabular}{|c|c|}
\hline \multicolumn{2}{|c|}{ Pamphlets } \\
\hline $\begin{array}{l}\text { Les Si et les Mais. Lettre à M. de la Porte } \\
\text { (anonyme, } 30 \text { juin) [se veut « impartiale»] }\end{array}$ & $\begin{array}{l}\text { Ch.M. de La Condamine, Les Quand, adres- } \\
\text { sés au sieur Palissot ( } 5 \text { juin) } \\
\text { Les Qu'est-ce? (anonyme, } 5 \text { juin) }\end{array}$ \\
\hline \multicolumn{2}{|c|}{ Préfaces } \\
\hline $\begin{array}{l}\text { Palissot, Lettre du Sieur Palissot [...] pour } \\
\text { servir de préface à la pièce ( } 5 \text { juin) }\end{array}$ & $\begin{array}{l}\text { A. Morellet, Préface de la comédie des } \\
\text { "Philosophes », ou la Vision de Charles } \\
\underline{\text { Palissot (29 mai) }}\end{array}$ \\
\hline \multicolumn{2}{|c|}{ Autres libelles (examens divers de la pièce) } \\
\hline $\begin{array}{l}\text { L. Coste d'Arnobat, Le philosophe, ami de } \\
\text { tout le monde, ou conseils désintéressés aux } \\
\text { littérateurs (sans date journalière) }\end{array}$ & $\begin{array}{l}\text { Lettre d'un original aux auteurs très origi- } \\
\text { naux (anonyme, mai 1760) }\end{array}$ \\
\hline $\begin{array}{l}\text { Conseil de lanternes, ou la véritable vision de } \\
\text { Charles Palissot (anonyme, } 10 \text { juillet) }\end{array}$ & $\begin{array}{l}\text { Petites Réflexions sur la comédie des } \\
\text { «Philosophes » (signé Candide le Cadet, } 19 \text { juin) }\end{array}$ \\
\hline Les Adieux (anonyme, 14 août) & Les Avis (anonyme, 26 juin) \\
\hline $\begin{array}{l}\text { I.H. de La Marche-Courmont, Réponse aux } \\
\text { différents écrits publiés contre la comédie des } \\
\text { «Philosophes » (11 septembre), réponse à } \\
\text { l'abbé G.-F. Coyer }\end{array}$ & $\begin{array}{l}\text { G.-F. Coyer, Discours sur la satire contre les } \\
\text { «Philosophes» (14 août) }\end{array}$ \\
\hline \multicolumn{2}{|c|}{ Poèmes } \\
\hline $\begin{array}{l}\text { C.J. Dorat, Épître à un ami dans sa retraite à } \\
\text { l'occasion des «Philosophes » et de } \\
\text { «L'Écossaise »(11 septembre) }\end{array}$ & $\begin{array}{l}\text { La vengeance de Thalie, poème } \\
\text { (anonyme, } 5 \text { juin) } \\
\text { J.-F. Marmontel, Un Disciple de Socrate, aux } \\
\text { Athéniens, héroïde ( } 7 \text { août) }\end{array}$ \\
\hline \multicolumn{2}{|c|}{ Recueil } \\
\hline & $\begin{array}{l}\text { Voltaire, Recueil des facéties parisiennes pour } \\
\text { le six premiers mois de l'an } 1760 \text { (à Genève) }\end{array}$ \\
\hline
\end{tabular}

Le premier coup est porté le 15 mai 1760 par le libraire Cailleau qui publie une fausse comédie en un acte intitulée Les Philosophes manqués, mettant aux prises des allégories qui se disputent sur la qualité de la pièce de Palissot : ainsi « Le Parterre » se querelle avec « La Comédie », « L'Intrigue », « L’Intérêt », le « Dénouement », la « Cabale », et bien sûr l'auteur lui-même des Philosophes. Olivier Ferret souligne qu'à travers cette mise en scène très explicite, se profile la crise de la comédie comme genre et comme manifestation d'une société corrompue.

Après cette querelle pseudo-littéraire, viennent deux pamphlets beaucoup plus violents, et surtout ad hominem. Le premier, dû à La Condamine, s'en prend à la mauvaise réputation de Palissot dans un petit texte rédigé sous la forme des «Quand », forme récemment mise à la mode par Voltaire qui avait semblablement 
attaqué Lefranc de Pompignan pour se moquer de son discours de réception à l'Académie française, particulièrement hostile aux philosophes (la forme en était une anaphore en «Quand »). Le texte de La Condamine reprend cette technique qui va faire fureur tout l'été : Les Quand, adressés au sieur Palissot, seront même le 5 juin republiés par Palissot lui-même, sans doute trop flatté de s'être attiré un libelle imité de Voltaire :

Quand on est auteur de Xarès et des tuteurs [...]

Quand on a gagné les Polichinelles de la littérature [...]

Quand on a prostitué sa femme à Nancy et à Paris $[\ldots]^{17}$.

L'abbé Morellet, qui a lu Les Quand de La Condamine et ne veut pas être en reste, publie le 29 mai, un degré plus haut dans la satire, une Préface de la comédie des "Philosophes » ou la Vision de Charles Palissot, où il commet l'imprudence de plaisanter sur la mauvaise santé de la protectrice de celui-ci, la princesse de Robecq, ce que Voltaire ne lui pardonnera pas, et ce pourquoi il sera d'ailleurs emprisonné pendant quelques semaines. Cette imprudence hélas discréditait les philosophes eux-mêmes plus encore que ne le faisait Palissot, les faisant passer pour de grossiers personnages incapables de se tenir en société, et prenant tous les risques pour régler quelques comptes personnels.

Palissot réplique à ce coup bas, le 5 juin, par une autre préface, peut-être déjà préparée de longue date et qui sera vendue sous le manteau, où il se réclame de la verve et de l'utilité satirique de Molière. Hélas, bien pire, il justifie la querelle qu'il fait aux philosophes en publiant une litanie de fausses citations prétendument tirées de leurs différentes œuvres ou bien sorties de leur contexte, visant à dénoncer l'immoralité et l'égoïsme prônés par la secte, ce que dénonçait déjà dans la comédie la scène du vol (un philosophe « fait les poches » d'un autre personnage). Voltaire, qui republiera toutes ces pièces en septembre sous le titre Recueil de Facéties parisiennes, aura la finesse de réattribuer, ou de corriger dans des notes spécifiques, toutes ces citations abusives, faisant preuve ici d'un véritable travail de pédagogie.

Le même jour, le 5 juin, paraît une réplique anonyme aux « Quand »: les "Qu'est-ce», offrant le même jeu de questions/réponses offensantes pour Palissot :

Qu'est-ce que ce poète, qui nous donne une si misérable comédie ? [...]

Qu'est-ce que ce titre qu'il prend, de plusieurs académies ? [...]

Qu'est-ce que cette idée, de faire marcher un pareil homme à quatre pattes $[\ldots]$ ? $^{18}$

Le même jour encore, le 5 juin, paraît une autre forme de pamphlet : épître, ou poème critique, la forme en est épique et dénonce en quelques pages et strophes la façon dont Palissot s'est fourvoyé : il s'agit de La Vengeance de Thalie, critique de la pièce des Philosophes, poème qui déplore la stérilité du sujet, la décadence du goût et l'absence d'ambition dont fait preuve la comédie, renvoyant à la crise que traverse le théâtre du temps :

17 Ch. Palissot de Montenoy, Les Philosophes, O. Ferret (dir.), pp. 102-105.

18 Ibidem, pp. 121-127. 
[...] On penserait qu'escroc vient de philosophie ;

Mais la vertu jamais n'enseigna l'infamie,

En épurant le cœur elle rend généreux :

Le sort du philosophe est d'être vertueux $[\ldots]^{19}$.

Un inventaire même rapide de ces textes fait apparaître la surreprésentation des échanges favorables aux Encyclopédistes, ainsi qu'une gradation générique qui veut qu'après des productions théâtrales parodiques, on voit s'accumuler des libelles en prose (textes plus polémiques), enfin des œuvres poétiques à valeur pacifiante ou bien marquées par une volonté de distanciation avec le conflit devenu avec le temps dérisoire. On observe également, du côté des défenseurs, l'intervention de seconds rôles philosophiques, tandis que les personnages visés (Diderot, Rousseau, Helvétius, Duclos) restent dans l'ombre. Quant aux arguments théoriques, après avoir souvent volé bas (en se concentrant sur l'immoralité prétendue des différents adversaires), ils s'enlisent dans la question de la vocation satirique ou non de la comédie, en invoquant Aristophane qui, selon certains, avec sa pièce Les Nuées, aurait précipité la chute de Socrate.

$* * *$

Il nous reste à examiner le rôle des échanges épistolaires dans la diffusion et l'extension du conflit " philosophique », que nous observerons ici de façon synthétique, à travers l'exemple contrasté de Diderot et de Voltaire. On reste en réalité très frustré devant la quasi absence de correspondance chez Diderot dans la première moitié de l'année 1760 . Une explication probable est que son amie intime, confidente et correspondante favorite, Sophie Volland, celle à qui il narre chacune de ses journées, a pu quitter Paris relativement tard en saison pour sa maison de campagne (à la mi-août), peut-être pour des raisons climatiques. Autre raison " climatique », mais médiatique cette fois : Diderot ne cherche-t-il pas à se faire oublier de ses autres correspondants pendant toute la période de mai à septembre ? Le fait est qu'on le retrouve à la campagne, chez $\mathrm{M}^{\mathrm{me}} \mathrm{d}$ 'Épinay puis chez le baron d'Holbach, se divertissant à écrire La Religieuse. On trouve toutefois une lettre essentielle de lui ${ }^{20}$, adressée au directeur de la librairie M. de Malesherbes le $1^{\text {er }}$ dimanche de juin 1760, indiquant qu'il n'est pas l'auteur de l'incendiaire " préface à la comédie des Philosophes ", soit de La Vision de Charles Palissot, qui enverra l'abbé Morellet à la Bastille; et ce dans des termes qui indiquent bien sa volonté de discrétion absolue, afin notamment de préserver l'Encyclopédie déjà bien compromise par la suppression de son privilège en 1759 :

19 Ibidem, p. 135.

${ }^{20}$ On sait aussi (par une allusion du patriarche à Louise d'Épinay, le 18 mai) qu'il aurait écrit à Voltaire, mais seul le début de cette lettre serait connu : «La seule vengeance qu'on puisse prendre de l'absurde insolence [...] » (Voltaire, Correspondance, t. V : 1758-1760, Th. Besterman (dir.), Gallimard, Paris 1980, p. 1537, note 2 de la page 908). 
Je n'ai point été à la pièce des Philosophes. Je ne l'ai point lue. Je n'ai point lu la Préface de Palissot, et je me suis interdit tout ce qui a trait à cette indignité. Loin de ces injures atroces, je ne serai point tenté de manquer à la promesse que je me suis faite et que je me suis tenue jusqu'à présent de ne pas écrire un mot de représailles. Quand les honnêtes gens veulent bien s'indigner pour nous, nous sommes dispensés de l'être. Je suis, avec un profond respect, etc. ${ }^{21}$

Fin août, il sort pour se distraire avec des amis à la Comédie-Italienne, voir $L a$ Guinguette et La Soirée des Boulevards (lettre à Damilaville, Ver., p. 206). Mais les autres courriers ayant trait au théâtre indiquent, chez Diderot, une préoccupation plus essentielle : il cherche à faire jouer sa propre pièce, Le Père de Famille, encore en lecture à la Comédie-Française au $1^{\mathrm{er}}$ septembre (lettre à $\mathrm{M}^{\mathrm{me}} \mathrm{d}^{\prime}$ Épinay, Ver., pp. 206-207, et à Sophie Volland, Ver., p. 209). Enfin, dans la même période, soit début septembre, il est subjugué par la tragédie que Voltaire fait jouer à son tour, Tancrède, dont il va faire l'examen et qu'il va inciter Sophie et ses sœurs à voir instamment ${ }^{22}$. Les 2, 5, 7, 10 septembre, il évoque également une «Épître du Diable à M. de Voltaire $»^{23}$, qu'on a pu croire de Palissot ; et enfin le Discours sur la Satire des " Philosophes », due à l'abbé Coyer, dont il juge que « c'est un ouvrage plein de modération et sur lequel il n'y a eu ici qu'un jugement ». Ceci nous prouve que Diderot, de façon constructive et non polémique, tente de placer sa réflexion sur un autre terrain, à savoir la possibilité de fonder un nouveau théâtre sur des bases esthétiques complètement différentes. Ce qui le ravit en effet, c'est (dans cette confidence à Sophie Volland du 5 septembre) l'estime intellectuelle que semble lui porter Voltaire : " De Voltaire avait lu à Grimm son Tancrède, lorsque celui-ci était à Genève; et il lui disait à propos des choses simples et des tableaux, "Vous voyez, mon cher, que j'ai fait bon usage des préceptes de votre ami" [ceux publiés dans les Entretiens sur le Fils Naturel et le Discours de la poésie dramatique, 1758] $»^{24}$. Hélas Le Père de Famille sera rejeté, par prudence sans doute, par les Comédiens-Français, et ne sera joué que de façon confidentielle, en province. Il ne fera son retour sur la célèbre scène parisienne qu'en 1769.

21 D. Diderot, Correspondance, [dans :] Euvres, t. V, L.Versini (dir.), Laffont, Paris 1997, p. 201. Désormais abrégé en Ver.

22 Lettres à Sophie Volland, les 2, 5, 10, 20 septembre 1760, [dans :] D. Diderot, Lettres à Sophie Volland : 1759-1774, M. Buffat et O. Richard-Pauchet (dir.), Non Lieu, Paris 2010 (désormais abrégé $L S V$ ). Voir aussi M. Buffat, « Diderot devant le théâtre de Voltaire », Recherches sur Diderot et sur l'Encyclopédie 47, 2012, pp. 119-134, ainsi que O. Richard-Pauchet, « Diderot et les hommes de théâtre », [dans :] Diderot. Théâtre. Musique, P. Frantz, R. Legrand et S. Marchand (dir.), Actes du colloque international organisé les 6-8 juin 2013 à l'Université Paris-Sorbonne [à paraître aux Presses universitaires de la Sorbonne].

23 Épître du Diable à M. de Voltaire, Claude-Marie Giraud, 1760.

${ }^{24} L S V$, p. 116. Diderot en revanche reconnaît la malfaisance de Fréron, évoqué en ces termes : « $\mathrm{M}^{\mathrm{me}}$ d'Épinay reçoit des lettres charmantes de De Voltaire. Il disait dans une des dernières que le diable avait assisté à la première représentation de Tancrède sous la figure de Fréron, et qu'on l'avait reconnu, à une larme qui lui était tombée des loges sur le bout du nez et qui avait [fait] pish, comme sur un fer chaud» (LSV, p. 129). 
Voltaire, à l'inverse, aura suivi l'événement pas à pas, jour après jour ${ }^{25}$, en rendant compte abondamment à tous ses correspondants, et adoptant selon chacun un ton soit partisan, soit détaché, soit ironique, soit carrément hostile, selon une ambiguïté savamment ménagée au gré de ses intérêts, qui sont avant tout de protéger sa propre carrière théâtrale tout en s'attirant le bénéfice de la cause intellectuelle portée par les Philosophes. Ainsi, tout à tour il jouera lui-même divers personnages épistolaires, tels :

\section{L'INDIGNÉ}

Le 25 avril, il s'afflige auprès de d'Alembert qui lui apprend le projet de Palissot, tout en déplorant la division des Philosophes, qui fait leur perte, et le masochisme de Rousseau : « C'est grand dommage que Jean-Jacques se soit mis tout nu dans le tonneau de Diogène » (Best., pp. 877-878) ${ }^{26}$. Le même jour, il essaie d'en savoir plus auprès de $\mathrm{M}^{\mathrm{me}} \mathrm{d}$ 'Épinay : « On dit que Diderot, dont le caractère et la science méritent tant d'égards, est violemment attaqué dans cette farce » (Best., p. 885). Auprès de d'Argental, diplomate chargé des relations de la cour auprès de la Comédie-Française, il commence un travail de lobbying et d'information, lui demandant « s'il est vrai qu'on joue une pièce contre les philosophes dans lequel on représente Jean-Jacques marchant à quatre pattes » (Best., p. 888). Avec Saurin, le 5 mai, il joue l'ignorant pour en savoir plus : « J'ignore si on a joué la farce contre les philosophes » et déplore leur désunion (« vous êtes plus fous que les Athéniens », Best., p. 895). Devant Jacques Lacombe, auteur dont il reçoit le livre et qu'il ne connaît pas, il monte sur ses grands chevaux : « Je suis aussi indigné que vous [...]. J'ai peine à croire que notre nation légère, soit devenue assez barbare, pour approuver une telle licence » (Best., pp. 897-898). Avec d'Argental, le 11 mai, il est beaucoup plus direct : «Est-il possible que $\mathrm{M}^{\mathrm{me}}$ de La Marck ait protégé si vivement une si infâme entreprise »(Best., p. 900). Le 18 mai, à nouveau la thèse de la désunion reparait dans une lettre à $\mathrm{M}^{\mathrm{me}} \mathrm{d}$ 'Épinay : «Au reste il est bon que ces pauvres philosophes s'aident mutuellement, comme les premiers chrétiens priaient Dieu les uns pour les autres » (Best., p. 908).

\section{LE RIEUR ET LE CYNIQUE}

Très vite, l'intérêt à la fois idéologique et financier de publier un recueil de toute cette querelle des libelles se fait jour, et de tirer ainsi les marrons du feu.

25 Nous ne dépouillons ici que le courrier du mois de mai, déjà excessivement abondant.

${ }^{26}$ Nos références renvoient ici à Voltaire, Correspondance, t. V : 1758-1760 (désormais abrégé Best.). 
À son fidèle ami Thieriot le 19 mai, il demande : « Je prie mon ancien ami de me faire avoir les $s i$, et les mais, et les pourquoi. Cela pourra faire un petit recueil à faire pouffer de rire ; on m'a envoyé les qui et les quoi. J'ignore quelle est la bonne âme qui a ainsi vengé les pauvres philosophes [...]. Ce monde est une guerre, celui qui rit aux dépens des autres, est le victorieux » (Best., p. 909). Même chose à $\mathrm{M}^{\mathrm{me}}$ d'Épinay, le même jour : « Ma belle philosophe, ces qui et ces quoi, qu'on m'envoie, m'ont amusé. Il faut rire de tout; il n'y a que ce parti-là de bon. On parle des $s i$, des mais, et des pourquoi. Il faut que quelque bonne âme fasse les comment!»(Best., pp. 900-910).

Auprès d'un ami demeurant en Allemagne, Élie Bertrand, il commence le 20 mai son travail de colporteur international de nouvelles, sans avoir vu la pièce ni même compris clairement l'intrigue : «On a joué [moqué] Jean-Jacques Rousseau à Paris et on l'a fait marcher à quatre pattes »(Best., p. 911)27. À un ami (le marquis de Thibouville) qui le complimente sur Tancrède qu'il lui a fait envoyer, il rétorque assez justement le 20 mai : « La guerre des auteurs est celle des rats et des grenouilles. Cela ne fait de mal à personne. Jansénistes, molinistes, convulsionnaires, Jean-Jacques voulant qu'on mange du gland, Palissot monté sur Jean-Jacques allant à quatre pattes $[. .$.$] , tout cela empêche qu'on ne soit trop$ occupé des désastres de nos armées et de nos flottes, et de nos finances » (Best., p. 912). Même commentaire à d'Alembert le lendemain, en insistant sur la maladresse des philosophes : " Jean-Jacques s'est rendu ridicule en voulant qu'on mangeât du gland, et en écrivant contre la comédie après avoir fait des comédies » (Best., p. 913). À $\mathrm{M}^{\mathrm{me}}$ Dupin de Tournay, le 22 mai, il défend encore la même thèse : «Les petites guerres intestines qu'on se fait à Paris sont aussi plates, que nos aventures sur terre et sur mer sont malheureuses [...]. Le goût de la campagne augmente encore le bonheur de ma retraite. Je n'y marche pas à quatre pattes, je crois qu'on peut être philosophe avec les aisances de la vie» (Best., p. 916).

\section{L'INTÉRESSÉ PUIS L'ENGAGÉ}

À partir du 25 mai, Voltaire commence à défendre ses intérêts de dramaturge en plaidant pour faire accepter, par l'intermédiaire de d'Argental, le manuscrit de L'Écossaise auprès des Comédiens-Français, texte qu'il fait passer pour une traduction ( « si vous avez la probité et le courage de faire jouer ce bon pasteur Hume », Best., p. 917). Surtout il intrigue (exploitant un conseil de d'Alembert) pour transformer en avantage le retard pris par une autre de ses tragédies, Médime, l'actrice Clairon étant tombée malade : «Très bonne idée, excellente idée de

27 Voir aussi la lettre à Jean-Robert Tronchin du 2 juin : « Rien n'est plus beau à présent que votre pays. Comptez que les billets de confession, les convulsions, les remontrances, et Rousseau Jean-Jacques marchant à quatre pattes sur le théâtre de Paris $[\ldots]$ ne valent pas nos charmants paysages » (Best., pp. 927-928). 
reculer Médime. Elle n'en vaudra que mieux. On aura le temps de la coiffer [répéter, peaufiner], elle ne paraîtra point après l'infamie contre les philosophes, et j'aurai la gloire de n'avoir pas voulu que les comédiens profitassent de ma pièce pour de l'argent au déshonneur de la nation » (26 mai, Best., p. 917) ${ }^{28}$. Voltaire, par ses relations, montre qu'il est au-dessus de la mêlée, connaissant à fond tous les grands qui protègent ou attaquent les deux partis : «M. de Choiseul est-il bien affligé de la maladie de $\mathrm{M}^{\mathrm{me}}$ de Robecq ? Je la tiens morte. C'est la maladie de sa mère. C'est bien dommage, mais pourquoi protéger Palissot ? Hélas, M. de Choiseul protège aussi ce Fréron [...]. Il ne connaît pas Fréron, c'est un monstre, mais un monstre dont je ne fais que rire » (Best., p. 918). Le 29 mai, il revient avec François de Chennevières à des idées plus littéraires : " La comédie autrefois riait du ridicule, aujourd'hui elle dit de grosses injures. Dans quel siècle vivons-nous? »(Best., p. 925). Le même jour, ayant reçu de toutes parts les libelles «monosyllabiques », il se plaint : "Au reste je ne sais pas pourquoi on me fourre dans toutes ces querelles, moi laboureur, moi berger, moi rat retiré du monde dans un fromage de suisse !» (à Thieriot, Best., p. 924).

Bientôt il se sent obligé d'entrer dans la mêlée. Le 31 mai, il doit fournir à d'Alembert des preuves de sa loyauté, ayant été en relation avec Palissot dans les années précédentes : " [après une énumération de points] $3^{\circ} \mathrm{M}$. Palissot de Montenoy et M. Patu vinrent aux Délices il y a plus de deux ans. M. Palissot me montra une pièce qu'il avait fait jouer à Lunéville ou à Nancy, et qui n'était point celle qu'il vient de donner à Paris. Je l'exhortai à ne point blasphémer contre la philosophie. $4^{\circ} \mathrm{Il}$ m'écrivit deux ou trois fois. $5^{\circ} \mathrm{Il}$ m'a donné un petit coup de patte dans sa comédie au sujet de Socrate. $6^{\circ}$ Il ne m'a pas envoyé sa pièce " (Best., pp. 925-926). Ce besoin soudain d'allégeance montre que Voltaire sent le vent tourner et pourrait se voir à son tour convaincu de complicité avec l'ennemi. D'ailleurs, Palissot lui envoie enfin sa pièce, et le voilà qui se fend d'une longue épître pleine de sévérité et de remontrances («Ayez la bonté de vous préparer à une réponse longue ; les vieillards aiment un peu à babiller », Best., pp. 930-934).

Cette correspondance passée, présente et à venir avec Palissot lui vaudra les sarcasmes de Diderot au mois d'octobre, dans une lettre à Sophie Volland où celui-ci fait le point sur toute cette affaire, égratignant et apaisant ici et là les passions terrestres qui ont présidé à ce déchaînement médiatique :

Les Facéties sont un recueil des impertinences de l'année 1760 que de Voltaire a fait imprimer à Genève et qu'il a grossi de quelques autres. La Vision y est, mais on en a supprimé les deux versets

28 Voir la lettre suivante à d'Alembert, du 26 mai, Best., p. 918 ; et à Thieriot, du 26 mai, Best., pp. 919-920 : « Je reçois la bêtise qu'on a jouée à Paris. J'en lis deux pages, je m'ennuie et je vous écris [...]. J'avais déjà retiré ma guenille tragique, quand Clairon est tombée malade, j'ai déclaré que je ne voulais rien donner à un théâtre où l'on a joué la raison et mes amis. Il m'est d'ailleurs très égal qu'on joue des pièces de moi, ou qu'on n'en joue pas. Je n'attends nulle gloire de ces performances. L'intérêt n'y a point de part puisque je donne le profit aux comédiens. MM. D'Argental font ce qu'ils veulent pour s'amuser ». 
de $\mathrm{M}^{\mathrm{me}}$ de Robecq ; et voilà, où je me trompe fort, la raison pour laquelle l'édition a été faite. Peutêtre aussi l'envie d'expier un peu la honte du commerce épistolaire avec Palissot y est-elle entrée pour quelque chose. Il a apostillé les lettres de Palissot de petites notes très cruelles. Il y a six mois qu'on s'étouffait à la comédie des Philosophes. Elle est au fond de l'abîme qui reste ouvert aux productions sans mœurs et sans génie; et l'ignominie est restée à l'auteur. Que le mot du philosophe athénien [Socrate] est beau ! Il disait à ceux qui le plaignaient : Ce n'est pas moi, c'est Anite et Mélite $^{29}$ qu'il faut plaindre. S'il fallait être à leur place ou à la mienne, balanceriez-vous ? Combien de circonstances dans la vie où l'on se consolerait de la même manière ? ${ }^{30}$

Mais on pourrait aussi donner, à l'inverse, le mot de la fin à $\mathrm{M}^{\mathrm{me}} \mathrm{du}$ Deffand, amie intime de Voltaire devenue fort conservatrice et qui, vexée de ne plus recevoir de ses lettres, reprend la plume pour l'occasion : «Voilà $\mathrm{M}^{\mathrm{me}}$ de Robecq morte, mais elle a trop tardé : six mois plus tôt nous auraient épargné une immensité de mauvais ouvrages » $(5 \text { juillet } 1760)^{31}$. Façon de suggérer que toute cette querelle n'a existé que pour flatter le pouvoir des Grands, qui ont instrumentalisé les Philosophes comme les anti-Philosophes, à seule fin de détourner l'opinion publique de réalités plus sordides : «D'ailleurs Monsieur, soyez sûr qu'il n’y a rien de plus ennuyeux que tous ces écrits et tous leurs auteurs; des cyniques, des pédants, voilà les beaux esprits d'aujourd'hui ; votre nom ne devrait jamais se trouver dans leurs querelles $»^{32}$.

29 Emprunt à Xénophon, [dans :] Apologie de Socrate, 29 (<http://remacle.org/bloodwolf/historiens/xenophon/apologie.htm> [consulté le 19.03.2020]). C'est Socrate qui parle. Anite et Mélite sont des accusateurs de Socrate lors de son procès.

${ }^{30}$ Lettre de Diderot à Sophie Volland, du 26 octobre 1760 (LSV, p. 187). À Voltaire, il écrira avec la même distance et la même hauteur de vue qu'il n'a « jamais lu ni les Petites Lettres sur de grands philosophes, ni cette satire dramatique où l'on me traduit comme un sot et un fripon; ni ces préfaces où l'on s'excuse d'une infamie qu'on a commise, en m'imputant tant de prétendues méchancetés que je n'ai pas faites et des sentiments absurdes que je n'eus jamais » (Ver., p. 332). Pour d'autres considérations sur Palissot et sa mauvaise réputation, voir H. Guénot, « Palissot de Montenoy : un "ennemi” de Diderot et des philosophes ", Recherches sur Diderot et sur l'Encyclopédie 1, 1986, pp. 59-63.

${ }^{31} \mathrm{M}^{\mathrm{me}}$ du Deffand, Cher Voltaire. La Correspondance de Madame du Deffand avec Voltaire, I. Vissière et J.-L. Vissière (dir.), Des Femmes - Antoinette Fouque, Paris 1987, pp. 74-75.

32 Ibidem. Cette vision conservatrice, mais également critique et distanciée du conflit, se retrouve dans d'autres versions à caractère pédagogique qui suivront Les Philosophes de Palissot. Ainsi cette comédie destinée à la jeunesse des collèges de Jésuites, intitulée Le Philosophe pyrrhonien (1761), montre les méfaits d'un philosophe trop sceptique, voire misanthrope (voir N. Brucker, « Tyrannie philosophique et pédagogie du rire dans le théâtre de collège : le cas du Philosophe pyrrhonien (1761) », [dans :] Gouvernement des hommes, gouvernement des âmes. Mélanges offerts à Charles Brucker, V. Bubenicek et R. Marchal (dir.), Presses universitaires de Nancy, Nancy 2007, pp. 101-110). 


\title{
LES PHILOSOPHES BY PALISSOT: THE QUARREL AND EPISTOLARY PASSIONS WHICH FOLLOWED THE PLAY
}

\author{
Summary
}

Les Philosophes, a French comedy by Palissot, a protégé of the royal minister Choiseul, was produced in Paris in May 1760 with the intention of discrediting Diderot and the Encyclopaedists. That summer it became a huge success, mainly due to the performance of an actor walking on stage on all fours (intended to denigrate Rousseau's reputation).

The purpose of the present article is to show, through the study of some of the lampoons and letters exchanged at that time, how the affair eventually tarnished the reputation of both parties, and how only the clever Voltaire emerged victorious from this heated debate.

Key words: Palissot, Diderot, Voltaire, Encyclopaedists, philosophers, satirical comedy. 